\title{
High-power heating of spherical tori by use of magnetic reconnection
}

\author{
Yoshinobu Ueda ${ }^{1 *}$ and Yasushi Ono ${ }^{2}$ \\ ${ }^{1}$ Department of Electrical Engineering, University of Tokyo, Tokyo 113-8656, Japan \\ ${ }^{2}$ High Temperature Plasma Center, University of Tokyo, Tokyo 113-8656, Japan
}

(Received June 21, 2000; Revised March 30, 2001; Accepted May 3, 2001)

\begin{abstract}
A novel high-power heating experiment of spherical tori (ST) has been developed in the TS-3 reconnection device using magnetic reconnection of merging STs. This method enables us to inject whole magnetic and thermal energies of a colliding ST into a target ST within short reconnection time. The maximum heating power of 15 MW was obtained in our low-field $(0.03-0.08 \mathrm{~T})$ and small-scale $(R<0.2 \mathrm{~m})$ merging experiment. This heating energy is provided mostly by anomalous ion heating effect of magnetic reconnection. The ion heating energy and the merging speed increases with decreasing the $q$-value ( $B_{t}$ component) of the ST. This heating effect causes the $\beta$-values of STs to increase by factor $2-3$.
\end{abstract}

\section{Introduction}

The TS (Tokyo Univ. Spherical Torus) experimental group has been investigating various magnetic reconnection phenomena of Spherical Tori (STs: spherical tokamak, spheromak and compact RFP) and their applications, using the TS-3 merging device. Its main objectives are (1) 3-D investigation of reconnection and its applications, (2) comparisons of various STs in a single device and (3) slow formation of FRC by use of spheromak merging and its application to ultra-high- $\beta$ ST formation. The high-power heating of ST is one of the major applications of magnetic reconnection to fusion plasmas (Ono, 1993). As shown in Fig. 1, the produced ST is collided with the target ST in the axial direction. Reconnection of their magnetic field lines is expected to heat plasma through its anomalous ion heating effect (Ono, 1996). This heating method is unique, because whole magnetic/thermal energy of the colliding ST is used for heating of the target ST within short merging/reconnection time (Ueda, 1999). Unlike the other heating methods, it will generate GW-order heating power easily in the present largescale tokamak experiments and is expected to be a future attractive high-power heating method of STs. Our TS-3 device can produce various STs with wide range of $q$-value from zero to 30 and then can merge them together. This paper addresses two important issues on the high-power heating characteristics of magnetic reconnection: (1) how its heating and reconnection characteristics depend on the $q$-value of the merging STs, (2) how this heating changes the ST equilibria, especially in terms of their beta $(\beta)$ values. The fast merging of low- $q$ ST realized the maximum heating power of $15 \mathrm{MW}$ in our initial experiment and increased $\beta$ of the target ST by factor $\approx 2-3$, revealing the high- $\beta$ properties of

\footnotetext{
*Present address: Meidensha Corporation, Tokyo 103-8515, Japan.
}

Copy right (c) The Society of Geomagnetism and Earth, Planetary and Space Sciences (SGEPSS); The Seismological Society of Japan; The Volcanological Society of Japan; The Geodetic Society of Japan; The Japanese Society for Planetary Sciences.
ST equilibria.

\section{Experimental Setup}

Our TS-3 merging device enables us to axially collide and to merge two STs with wide range of $q$-value from 0 to 30. As shown in Fig. 2, the TS-3 merging device has two poloidal field (PF) coils and two sets of eight electrode pairs for two ST formations in a cylindrical vacuum vessel with length of $1 \mathrm{~m}$ and diameter of $0.8 \mathrm{~m}$. These coils were used to inject arbitrary amount of toroidal and poloidal fluxes into the STs. Right after their formation, the STs have major and minor radii of $0.2 \mathrm{~m}$ and $0.14 \mathrm{~m}$. Reversed currents $I_{\text {acc }}$ of the two PF coils were used to increase the plasma colliding force as well as the reconnection speed. Using the center torus coil current $I_{t f c}$, the arbitrary toroidal field $B_{t, \text { ext }}$ was applied to the STs, varying toroidal field $B_{X}$ ( $=B_{t, \text { ext }}+B_{t, \text { in }}$ ) of the ST continuously from low- $q$ RFP region to high- $q$ tokamak region. As $I_{t f c}$ was increased from $-5 \mathrm{kA}$ to $50 \mathrm{kA}$, the $q$-value was observed to increase from the RFP regions $\left(q_{0} \sim 0.1\right)$, through the spheromak region to the tokamak region $\left(q_{0}>1\right)$.

Radial profile of ion temperature $T_{i}$ was measured on the midplane by use of a Doppler broadening of $\mathrm{H}_{\beta}$ and $\mathrm{C}_{\mathrm{II}}$ lines. The 2-D array of magnetic probe is located on the $r-z$ plane of the vessel to measure the poloidal and toroidal magnetic field profiles of the merging plasmas. An electrostatic probe is inserted at $z=0$ to measure radial profiles of electron temperature $T_{e}$ and density $n_{e}$.

In the present operation, the initial merging plasmas had the poloidal magnetic field $B_{p} \sim 0.05 \mathrm{~T}$, ion and electron temperatures $T_{i} \approx T_{e} \sim 5-10 \mathrm{eV}$ and density $n_{e} \sim$ $10^{20} \mathrm{~m}^{-3}$.

\section{Experimental Results}

\subsection{High power heating effect of merging}

Figures 3 show poloidal flux contours of the merging STs and those of a single ST for comparison. For simplicity, flux 


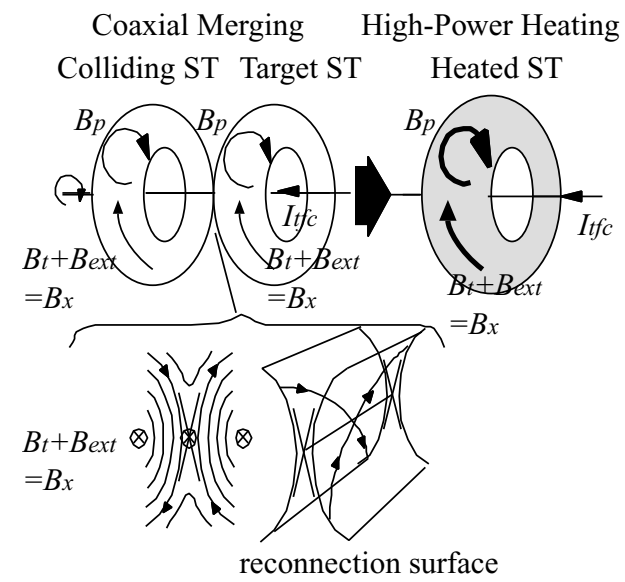

Fig. 1. Plasma heating scheme of compact tori by use of merging (magnetic reconnection) effect.

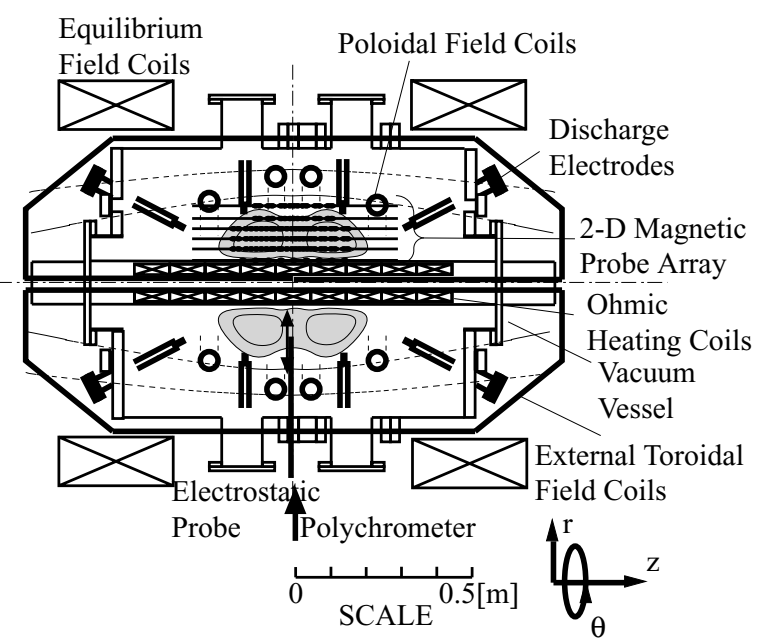

Fig. 2. The TS-3 merging device.

of the colliding ST was set equal to that of the target ST in the merging case. Electrode discharge initiated at $t=0$ and the two STs were observed to merge together during $t=45$ $\mu$ sec-55 $\mu$ sec.

During this merging phase, the magnetic reconnection was found to increase ion temperature of the target ST by factor 2-10. Figures 4 show time evolutions of (a) ion temperatures and (b) electron temperatures and (c) densities of single and merging spheromaks $\left(B_{x 0}=0\right)$. While electron temperature and density stayed almost constant, ion temperature $T_{i}$ increased significantly from $10 \mathrm{eV}$ to $50 \mathrm{eV}$ within the short reconnection (merging) time of $15 \mu \mathrm{sec}$. As shown in (Ono et al., 2001), the magnetic reconnecrtion was found to convert the poloidal magnetic energy into ion outflow energy. The outflow speed almost equal to the ion thermal speed was observed to increase inversely with $B_{x 0}$ component.

It was observed that the large increase in ion temperature provided large heating energy to the merging toroids. During this merging phase, the thermal energy of the target ST (a) Single ST

$B x 0=35[\mathrm{mT}]$
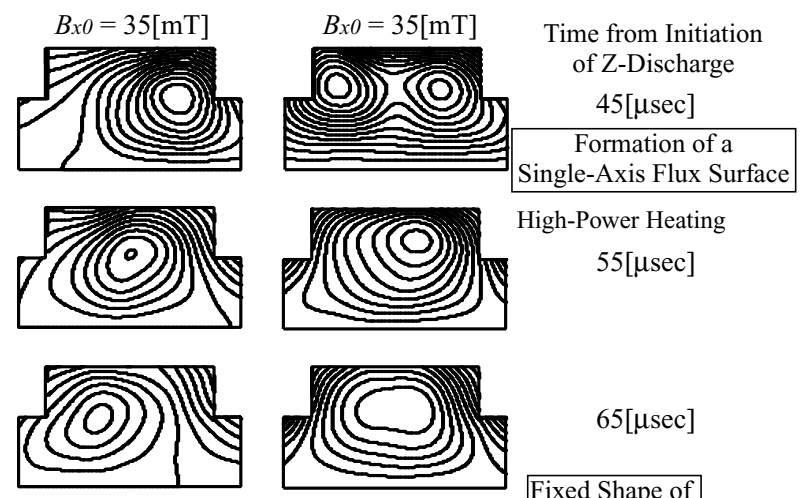

High-Power Heating

$55[\mu \mathrm{sec}]$

Contour Spacing $0.5[\mathrm{mWb}]$

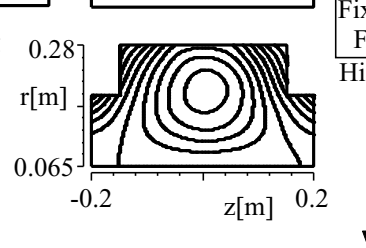

$65[\mu \mathrm{sec}]$

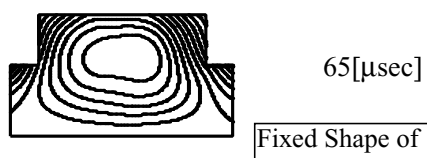

Flux Surface

High- $\beta$ Equilibrium

$75[\mu \mathrm{sec}]$

Resistive Decay

$(\sim 40[\mu \mathrm{sec}])$

Fig. 3. Time evolutions of poloidal flux surfaces. (a) Single ST, (b) merging $\mathrm{ST}\left(B_{x 0}=35 \mathrm{mT}\right.$, respectively).
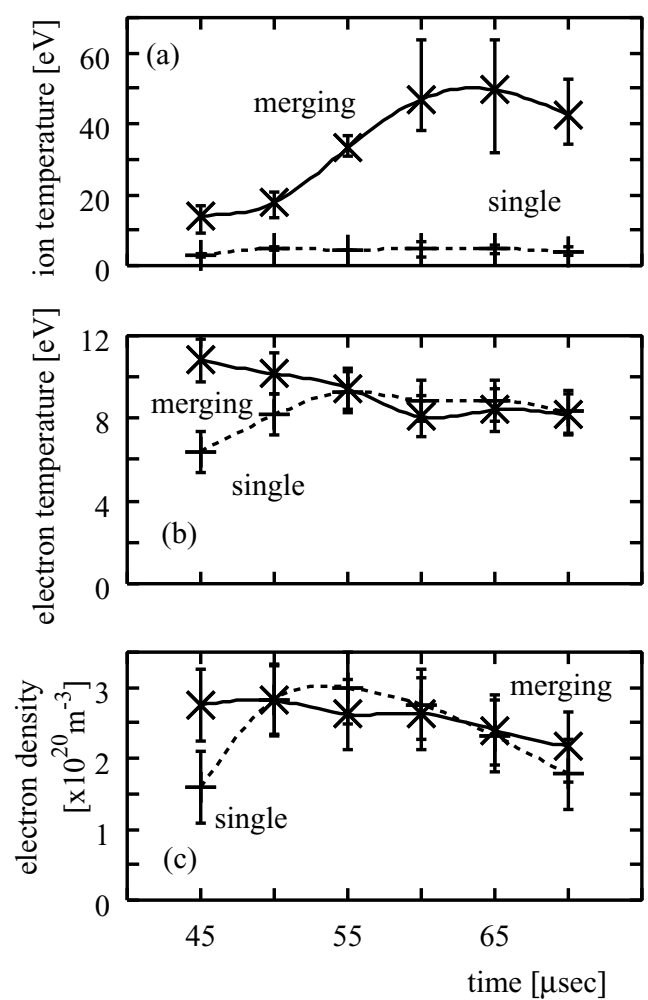

Fig. 4. Time evolutions of (a) ion temperatures, (b) electron temperatures and $(\mathrm{c})$ densities of spheromaks $\left(B_{x 0}=0\right)$. Solid lines indicate merging spheromaks and dashed lines indicate single spheromaks.

was found to increase by factor $2-10$. Figures 5 show thermal energies of merging spheromak $\left(B_{x 0}=0\right)(a)$ and those of spherical tokamak $\left(B_{x 0}=35 \mathrm{mT}\right)(\mathrm{b})$, based on measurements of $T_{i}, T_{e}$ and $n_{e}$. In this experiment, poloidal fluxes of initial STs were made constant (within 10\%). Since the sizes of merging toroids were kept the same, toroidal poloidal 
(a) Spheromak $(B x 0=0)$

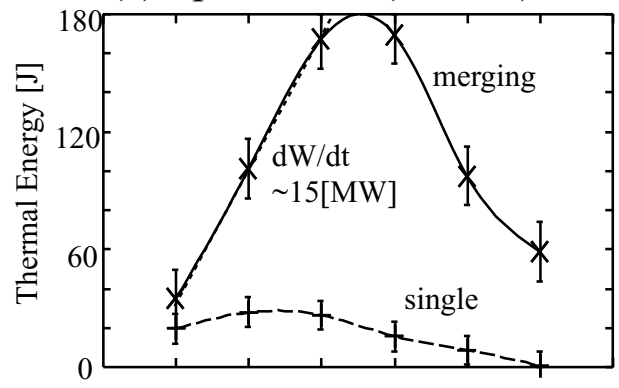

(b) high- $q$ ST $(B x 0=35[\mathrm{mT}])$

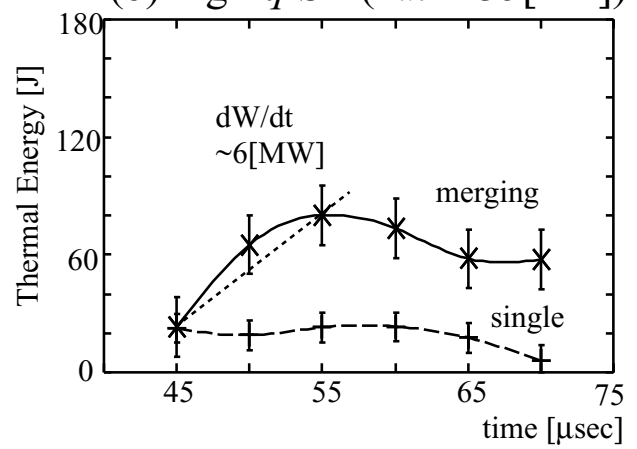

Fig. 5. Time evolutions of thermal energies of merging STs (solid lines) and single STs (dotted lines). The STs are (a) spheromaks $\left(B_{x 0}=0\right)$ and (b) high- $q$ STs $\left(B_{x 0}=35 \mathrm{mT}\right)$.

magnetic energies and plasma currents were also set constant for this comparison. The thermal energies of single spheromak and single tokamak are also shown for comparison, As shown in the following equation, those thermal energies were calculated inside the separatrix by assuming a parabolic thermal pressure profile $p=P_{0}\left(1-u^{2} / a^{2}\right)$.

$$
\begin{aligned}
W & =\int_{0}^{a} \int_{0}^{2 \pi} P_{0}\left(1-\frac{u^{2}}{a^{2}}\right) 2 \pi\left(R_{0}+u \cos \omega\right) u d \omega d u \\
& =\int_{0}^{a} P_{0}\left(1-\frac{u^{2}}{a^{2}}\right) 4 \pi^{2} R_{0} u d u \\
& =P_{0} \pi^{2} a^{2} R_{0} \\
& =\frac{1}{2} P_{0} V,
\end{aligned}
$$

where $P_{0}$ is a pressure at magnetic axis, $u$ is a distance from magnetic axis, $a$ is minor radius of the $\mathrm{ST}, R_{0}$ is major radius, $\omega$ is poloidal angle and $V$ is the volume of ST. Because external driving (push) force for merging was maximized in this experiment, merging time was less than 10 $\mu$ sec. Effect of transport loss was neglected because the energy confinement time of an ST (especially in high- $q$ case) is much longer than $10 \mu \mathrm{sec}$. In both cases, the thermal energies of the merging STs were observed to increase significantly right after they started merging. The maximum heating power of $15 \mathrm{MW}$ was obtained in the case of merging spheromak $\left(B_{x o}=0\right)$. This power was provided mostly by the ion heating effect of magnetic reconnection as shown in (Ono et al., 2001). Even if the flux conversion caused by injection of toroidal flux had some contribution to the ion heating, it ought to take place 20 microseconds after the

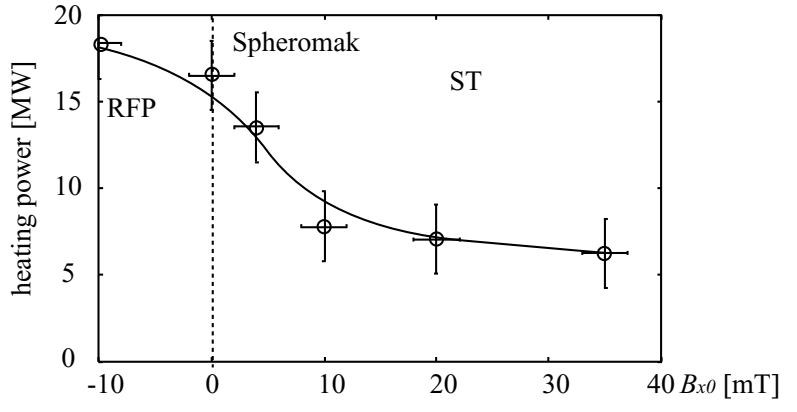

Fig. 6. Heating power of ST merging as a function of $B_{x 0}$.

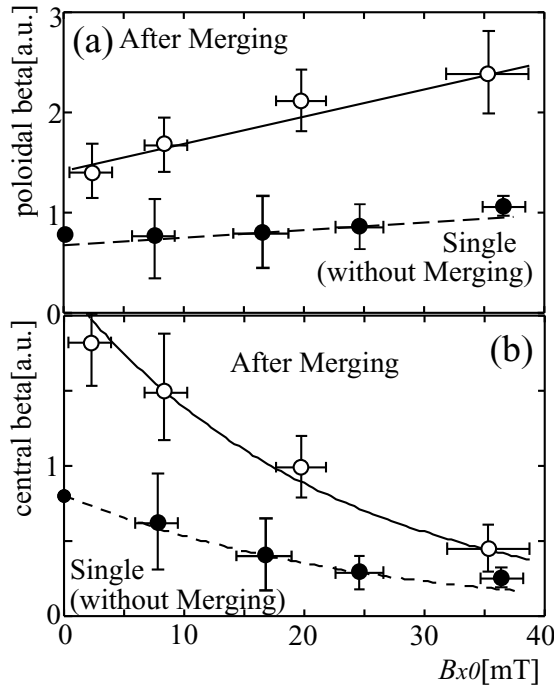

Fig. 7. $B_{x 0}$ dependencies of (a) poloidal beta $\beta_{p}$, (b) central beta $\beta_{0}$.

heating by merging itself. The flux conversion: poloidal flux amplification was observed to occur occurred about $20 \mathrm{mi}-$ croseconds after the completion of reconnection. The thermal energies of the merged STs were much larger than those of the single STs in both cases. It is considered that the large heating power (energy) was due to large anomalous resistivity observed in low $B_{x 0}$ (low- $q$ ) reconnection. The effective resistivity of the current sheet was observed to increase significantly when the current sheet width was compressed shorter than the ion Larmor radius (Ono et al, 2001). It is also noted that the heating energy increases with decreasing the $q$-value of the merging STs as shown in Fig. 6.

\subsection{High beta properties of STs}

Another question is how much of the injected heating energies are confined in those STs after the reconnection. As shown in Fig. 5, the thermal energy of the high- $q$ ST was observed to decay much more slowly than that of the spheromak. These results indicate that the ST with higher $q$-value has better confinement to sustain the large heating energy of merging. When the size of the colliding ST is the same as that of the target ST, poloidal flux was kept constant while toroidal flux is doubled based on the 2-D approximation. Merging low- $q$ STs was considered to relax into a stable equilibrium through flux conversion from toroidal 
to poloidal. This relaxation process causes the STs to lose large portion of thermal energies injected by the merging. It was observed that the high- $q$ STs can maintain the high$\beta$ equilibrium deviated from the Taylor state because of its flexibility of equilibrium.

The high- $\beta$ equilibrium was characterized by a hollow current profile and a reduction of the paramagnetic toroidal field. A high- $\beta$ current sheet was produced in the reconnection region and remains as a hollow current profile after the reconnection was over. This hollow $j_{t}$ profile was found to maintained until the decay of the ST. These two properties enable high- $\beta$ operation described as follows. The radial component of the MHD pressure balance equation is

$$
\frac{d p}{d r}=j_{t} B_{z}-j_{z} B_{t} .
$$

Equation (2) can be separated into the first and the second term because $j_{t}$ is calculated from $B_{z}$ and $j_{z}$ is calculated from $B_{t}$ when an $\mathrm{ST}$ is axisymmetric. The first term indicates that a hollow $j_{t}$ profile sustains a higher and broader thermal pressure than a peaked. The second term indicates that the pressure gradient increases with decreasing $j_{z}$ which induces paramagnetic toroidal field. These two effects enabled the merging STs to confine larger thermal energy than single STs with the same size of magnetic pressure.

Figures 7 show the poloidal beta $\beta_{p}$ and the central beta $\beta_{0}$ of the STs after merging and the single STs, as a function of $B_{x 0}$. It was clearly observed that the merging process increases $\beta_{p}$ and $\beta_{0}$ by factor $2-3$. It is noted that the $\beta_{p}$ increment increases with increasing $B_{x 0}$ ( $q$-value). It is interesting to check whether this tendency agrees with Troyon scal- ing or not. It is concluded that the high- $\beta$ confinement of CT improves with increasing its $q$-value, while the ion heating energy of merging increases with decreasing the $q$-value.

\section{Conclusions}

In summary, our TS-3 ST merging experiments demonstrated the novel high-power heating of merging STs for the first time. The maximum heating power of $15 \mathrm{MW}$ was obtained in our low-field $(0.03-0.08 \mathrm{~T})$ and small-scale $(R<$ $0.2 \mathrm{~m}$ ) experiment. This high-power heating is caused by the anomalous ion heating effect of magnetic reconnection. The ion heating energy and merging speed was observed to increase with decreasing $q$-value $\left(B_{x 0}\right)$ of merging toroids. However, the larger thermal energy was confined in the ST with higher $q$-value $\left(B_{x 0}\right)$. The final $\beta_{p}$ of the merging ST increases with its $q$-value $\left(B_{x 0}\right)$, suggesting that the high- $q$ ST has more flexibility to adjust its equilibrium to high- $\beta$ state.

\section{References}

Ono, Y., Experimental investigation of three-dimensional magnetic reconnection by use of two colliding spheromaks, Phys. Fluids B, 5, 36913701,1993

Ono, Y., Ion accerelation and ion heating in three-component magnetic reconnection, Phys. Rev. Lett., 76, 3328-3331, 1996.

Ono, Y., M. Inomoto, Y. Ueda, T. Matsuyama, and Y. Murata, Fast compression of a current sheet during externally driven magnetic reconnection, Earth Planets Space, 53, this issue, 521-526, 2001.

Ueda, Y., High-power heating experiment of spherical tokamaks by use of plasma merging, Trans. IEE Japan, 119-A, 1319-1323, 1999.

Y. Ueda (e-mail: kien@katsurai.t.u-tokyo.ac.jp) and Y. Ono 